\title{
Single-scatter Monte Carlo compared to condensed history results for low energy electrons *
}

\author{
C.T. Ballinger, D.E. Cullen, S.T. Perkins and J.A. Rathkopf \\ Lawrence Livermore National Laboratory, University of California, P.O. Box 808, L-18, Livermore, California 94550, USA
}

\author{
W.R. Martin and S.J. Wilderman \\ The University of Michigan, Department of Nuclear Engineering, Ann Arbor, Michigan 48109, USA
}

Received 27 November 1991 and in revised form 19 May 1992

\begin{abstract}
A Monte Carlo code has been developed to simulate individual electron interactions. The code has been instrumental in determining the range of validity for the widely used condensed history method. This task was accomplished by isolating and testing the condensed history assumptions. The results show that the condensed history method fails for low energy electron transport due to inaccuracies in energy loss and spatial positioning.
\end{abstract}

\section{Introduction}

The condensed history Monte Carlo methodology [1] is widely acknowledged to be inadequate for the simulation of low energy electron transport, especially in materials of high atomic number. A number of restrictive assumptions about electron scattering characteristics are necessary to make possible an analytical solution to the infinite-medium transport equation. Distributions describing electron characteristics after multiple collisions (multi-scatter distributions) are constructed from such solutions and serve as the heart of condensed history codes. In this paper a two-level approach is taken to quantify the errors inherent in the condensed history approach. First, conventional, condensed history multiscattering distributions in energy and angle are compared directly with analogous distributions generated with a single-scatter Monte Carlo code. This recently developed code directly simulates individual electron interactions. Such a comparison is, in essence, a numerical experiment once the validity of the single scatter code is accepted. Second, in the condensed history code, the conventional distributions are replaced by distributions constructed via a singlescatter Monte Carlo simulation. Experimentally verifi-

* This work was performed under the auspices of the U.S. Department of Energy by Lawrence Livermore National Laboratory under contract No. W-7405-Eng-48.

Correspondence to: J. Rathkopf, Lawrence Livermore National Laboratory, University of California, P.O. Box 808 Livermore, CA 94550 , USA. able quantities such as backscatter coefficients were calculated by the condensed history code using each set of distributions. We have identified three sources of error in the condensed history method; inaccuracies arise from the use of

(1) multiscatter angular deflection theory,

(2) multiscatter energy loss theory, and

(3) the straight flight path approximation.

It will be shown that correcting errors in the energy loss theory offers the greatest improvement over the condensed history method. Although the energy loss theory used in conventional condensed history calculations preserves the correct mean energy loss, the distributions about the mean are vastly different than single scatter results.

The following section describes and validates the single scatter Monte Carlo code. Sections 3 and 4 describe each level of the comparison approach alluded to above. Section 5 provides some discussion of the conditions under which condensed history fails and briefly describes a new method, the Response History Monte Carlo Method [2], that is a candidate to supplement condensed history calculations for low energies. This new method inspired the substitution of distributions described in section 4 .

\section{Single-scatter Monte Carlo}

Simulating individual electron scattering events using the Monte Carlo method is computationally intensive due to the extremely large cross sections for 
Coulombic interactions. Electron cross sections arc frequently five orders of magnitude larger than for photons of the same energy. Despite its computational shortcomings, single scatter (analog) Monte Carlo proves to be a very useful tool for testing the validity of the approximations used in condensed history. The code used in this study was developed at the Lawrence Livermore National Laboratory to utilize the recently developed cross section data base [3-5]. The data base includes information about impact ionizations, bremsstrahlung, and elastic scattering interactions for elements with atomic number ranging from 1 through 100 and energies from $10 \mathrm{eV}$ to $100 \mathrm{GeV}$. Most previous attempts to perform single-scatter Monte Carlo relied heavily on the continuous slowing down approximation (CSDA) for describing electron energy loss. The new cross section data base includes energy loss information for individual bremsstrahlung and impact ionization events which greatly reduces the dependence on the CSDA for the energy loss.

Cross sections are tabulated at various energics for elastic scatter, impact ionization, and bremsstrahlung interactions. Probability distributions functions (PDFs) describe elastic scattering angles for individual collisions. Mott's [6] elastic scattering cross sections are used for energies above $256 \mathrm{keV}$ whilc Rilcy [7] data is used for lower energies. The Mott and Riley data were extrapolated to encompass energies between $10 \mathrm{eV}$ and $100 \mathrm{GeV}$. The differential elastic scattering cross section includes scattering events with cosine between 0.999999 and -1 which rcsults in an accuratc representation of individual collisions. Energy loss PDFs are tabulated for bremsstrahlung and impact ionization events. Impact ionization data is given for every shell of the ground state target atom. Energy loss interactions grcatcr than $10 \mathrm{cV}$ are included in the differential bremsstrahlung and impact ionization data.

A restricted stopping power is used to determine the energy loss associated with excitation. This is accomplished by subtracting the ionization component from the total collisional stopping power. Data by Ashley et al. [8] is used for collisional stopping powers below $10 \mathrm{keV}$ while the U.S. National Bureau of Standards data [9] is used for higher energies. Energy loss by impact ionization is the dominant energy loss mechanism for keV-range electrons so applying the CSDA to the excitation component should not impact the accuracy of the results. Energy loss straggling in the analog Monte Carlo code is accounted for explicitly by considering individual impact ionization and bremsstrahlung interactions.

\subsection{Analog code benchmarking}

A wealth of experimental data exists for monoenergetic electron beams normally incident on various ma-

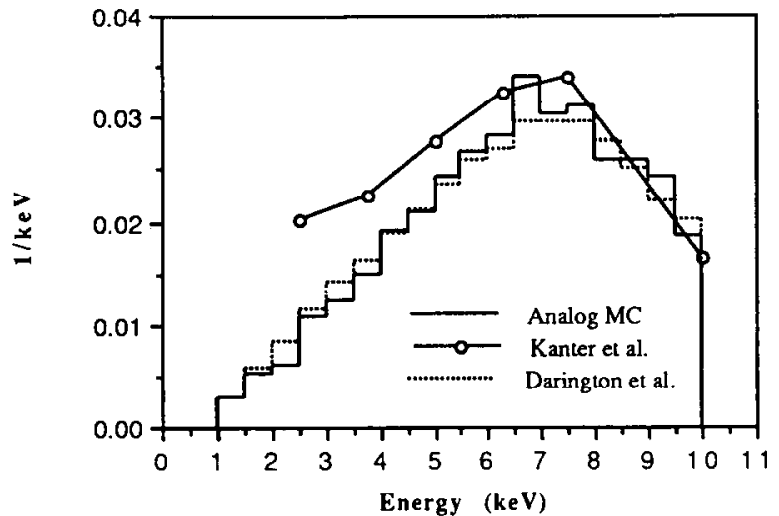

Fig. 1. Backscattered energy spectrum for $10 \mathrm{keV}$ electrons normally incident upon thick aluminum.

terials. Analog Monte Carlo calculations for transport in gold and aluminum were compared to experimental measurements to provide benchmarking. A cut-off energy of $1 \mathrm{keV}$ was established for all the Monte Carlo comparisons since condensed history codes typically do not track electrons below this energy. The differential backscatter energy spectrum for $10 \mathrm{keV}$ normally incident electrons on thick aluminum is shown in fig. 1 . Backscatter calculations are an excellent test for the analog Monte Carlo code since condensed history codes have historically had difficulties with this type of problem. The analog code compares well with the measurement by Darlington et al. [10] and Kanter et al. [11]. Similarly, good agreement is shown in fig. 2 for $20 \mathrm{keV}$ electrons normally incident on a thick gold target. The analog code works well for both high and low atomic number materials. As expected, condensed history results disagreed with the analog Monte Carlo and experimental results for these low energy backscatter problems so they were not included in the comparisons thus far.

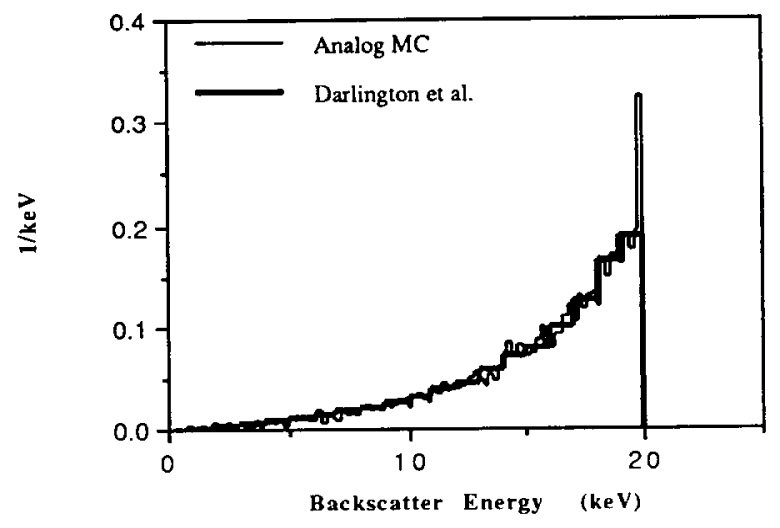

Fig. 2. Backscattered energy spectrum for $20 \mathrm{keV}$ electrons normally incident upon thick gold. 
The condensed history method works well for higher energies as demonstrated by the transmitted energy spectrum for $1 \mathrm{MeV}$ electrons exiting through a 0.15 $\mathrm{g} / \mathrm{cm}^{2}$ gold foil as shown in fig. 3. Results from the analog Monte Carlo code nearly reproduce the experimental values by Rester et al. [12] and are in concurrence with the condensed history codes, ETRAN and MCNP4 [13]. The ETRAN data was taken from the Rester article while MCNP4 (a new version of MCNP for neutron, photon, and electron transport) calculations were performed by the authors. The transmitted fraction calculated in the analog code for this problem was $0.379 \pm 1.9 \%$ which compares well with the 0.37 transmitted fraction predicted by both ETRAN and MCNP4. The experiment by Rester et al. quotes 0.43 as the transmitted fraction. The difference between the analog results and the experimental measurements is at least partially attributable to the lack of photonelectron coupling in the analog code. This difference will be reduced as energy decreases for bremsstrahlung energy loss is more prevalent for high energy electrons.

Backscatter calculations are interesting since they are especially difficult for condensed history calculations to reproduce. Backscatter percentages, as functions of energy, for electrons normally incident on gold and aluminum are shown in figs. 4 and 5 respectively. These figures reveal the energy range of validity for the condensed history codes, SANDYL [14] and MCNP4. The excellent agreement between the analog Monte Carlo code and experimental values by Neubert et al. [15] and Darlington ct al. shows that the analog Monte Carlo code is performing correctly. The condensed history methodology underestimates the backscatter percentage for energies below approximately $30 \mathrm{keV}$ in aluminum and below $80 \mathrm{keV}$ in gold. Several other comparisons, not shown here, for different encrgies

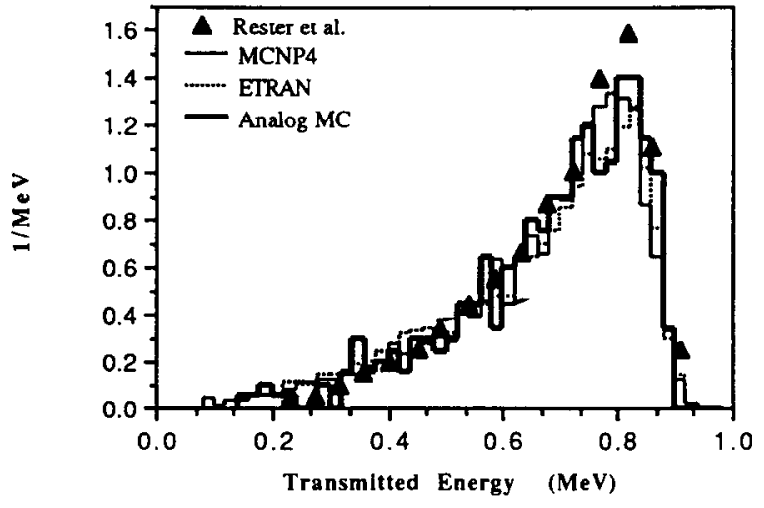

Fig. 3. Transmitted energy spectrum for $1 \mathrm{MeV}$ electrons normally incident upon $0.15 \mathrm{~g} / \mathrm{cm}^{2}$ of gold.

indicate that integrated and differential quantities measured experimentally are reproducible with the analog Monte Carlo code.

\section{Evaluation of multiscattering distributions}

The condensed history method was developed to approximate the cumulative effect of many collisions for high energy electrons. The cornerstone for the condensed history method is the assumption that the electron energy and direction can be updated by sampling from PDFs representing electron characteristics after a certain path-length has been traveled. Different theories exist for approximating the electron state after traveling a certain path-length and suffering multiple collisions. These multiscatter PDFs require assumptions about the electron scattering angle, energy loss, and spatial diffusion. These restrictive assumptions

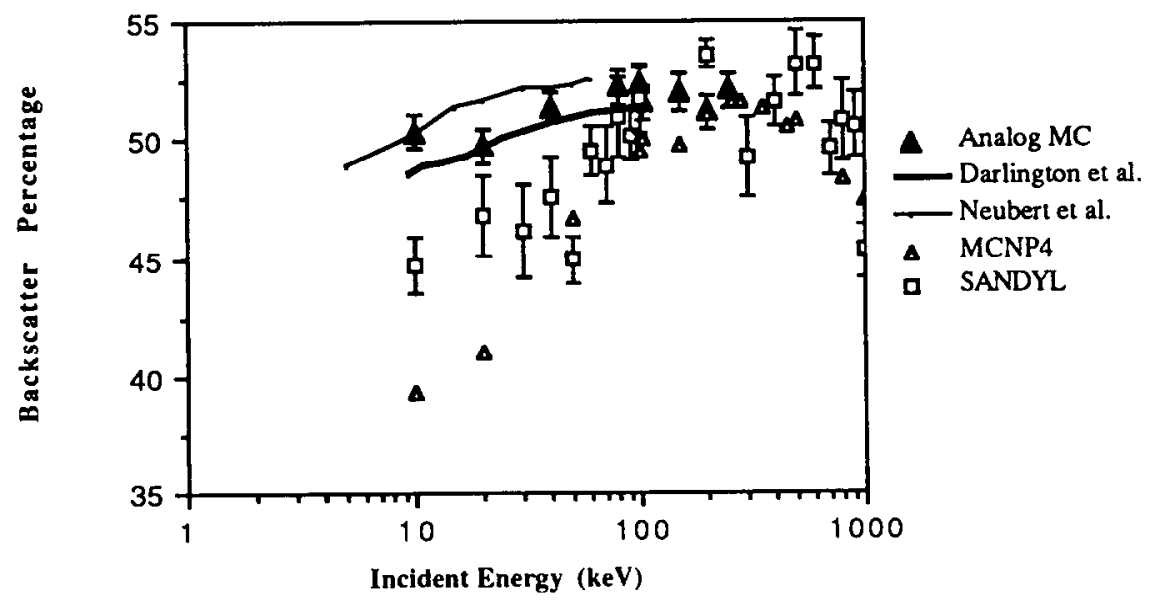

Fig. 4. Backscatter percentage for electrons normally incident on thick gold. 


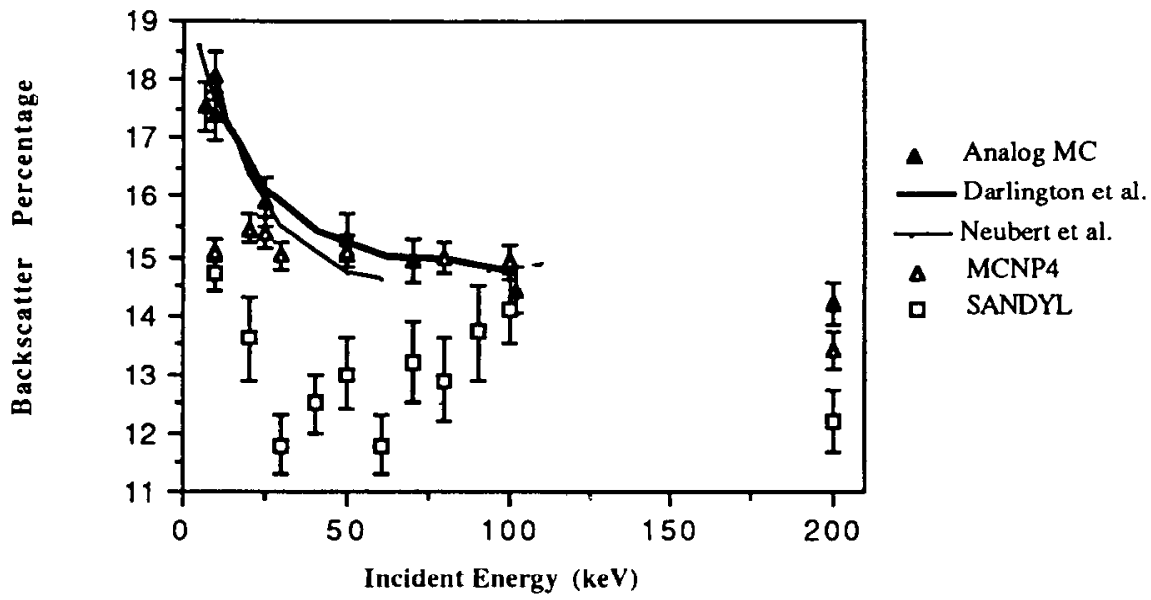

Fig. 5. Backscatter percentage for electrons normally incident on thick aluminum.

prove to be the downfall of the condensed history method for low energy electrons.

The multiscatter PDFs are calculated for a set of particular energies, $E_{i}$, before the electron simulation commences. The energy spacing is determined by assigning a number of energy steps, $N$, for the electron to lose half its initial energy, $E_{\text {init }}$,

$E_{i}=E_{\text {inil }}\left(\frac{1}{2}\right)^{i / N}$.

The average distance, $r_{i}$, traveled by an electron between $E_{i}$ and $E_{i+1}$ is calculated using the CSDA,

$r_{i}=\int_{E_{i+1}}^{E_{i}}\left(\frac{\mathrm{d} E\left(E^{\prime}\right)}{\mathrm{d} x}\right)^{-1} \mathrm{~d} E^{\prime}$.

The energy step is further divided into a number of substeps, $N_{\mathrm{SS}}$, such that the substep distance equals $r_{i} / N_{\mathrm{Ss}}$.

Multiscatter distributions are constructed to give the angular distribution of electrons that have traveled the substep distance. Ideally, the substeps are chosen small enough so that the actual trajectory of the electron can be replaced by a straight line segment. The deviation of the electron path from this straight line becomes substantial for certain energies which causes erroneous results in the condensed history calculation.

The number of collisions encountered by electrons while traveling an MCNP4 default substep size are given in table 1. For low enough energies, the number of collisions decreases to one or less which invalidates the multiple scattering assumption required for the derivation of some multiscatter distributions. Although in theory the Goudsmit-Saudersen multiscatter distribution [16] described in the following section is valid even for single collisions, such accuracy requires hundreds of Legendre expansion terms. The higher order terms pose numerical problems. The Molière distribution [17], another analytical multiscatter distribution, is generally considered invalid for less than 20 collisions.

Table 1

Average number of collisions after traveling an MCNP4 default substep distance

\begin{tabular}{|c|c|c|c|c|c|c|}
\hline \multirow{2}{*}{$\begin{array}{l}\text { Energy } \\
(\mathrm{keV})\end{array}$} & \multicolumn{3}{|c|}{ Aluminium } & \multicolumn{3}{|l|}{ Gold } \\
\hline & Elastic & Ionization & $\begin{array}{l}\text { Substep } \\
\text { (cm) }\end{array}$ & $\overline{\text { Elastic }}$ & Ionization & $\begin{array}{l}\text { Substep } \\
\text { (cm) }\end{array}$ \\
\hline 1 & 1.41 & 0.529 & $9.187 \times 10^{-8}$ & 1.02 & 0.641 & $2.821 \times 10^{-8}$ \\
\hline 2 & 1.95 & 0.685 & $2.725 \times 10^{-7}$ & 1.11 & 0.595 & $4.261 \times 10^{-8}$ \\
\hline 4 & 2.98 & 0.960 & $8.629 \times 10^{-7}$ & 1.52 & 0.632 & $1.046 \times 10^{-7}$ \\
\hline 8 & 4.79 & 1.51 & $2.834 \times 10^{-6}$ & 2.24 & 0.821 & $2.989 \times 10^{-7}$ \\
\hline 16 & 8.01 & 2.55 & $9.482 \times 10^{-6}$ & 4.15 & 1.16 & $9.139 \times 10^{-7}$ \\
\hline 32 & 13.72 & 4.08 & $3.170 \times 10^{-5}$ & 7.90 & 1.86 & $2.868 \times 10^{-6}$ \\
\hline 64 & 23.78 & 8.09 & $1.034 \times 10^{-4}$ & 15.32 & 3.10 & $8.908 \times 10^{-6}$ \\
\hline 128 & 42.77 & 14.84 & $3.180 \times 10^{-4}$ & 29.22 & 5.45 & $2.623 \times 10^{-5}$ \\
\hline 256 & 68.56 & 25.09 & $7.824 \times 10^{-4}$ & 48.39 & 8.80 & $6.221 \times 10^{-5}$ \\
\hline
\end{tabular}




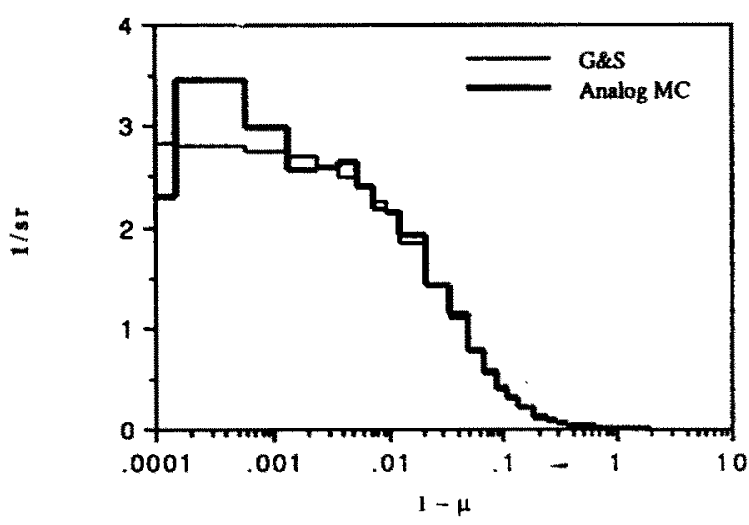

Fig. 6. Multiscatter angle distributions for $256 \mathrm{keV}$ electrons after a path-length of $6.22 \times 10^{-5} \mathrm{~cm}$ in gold.

\subsection{Multiscatter angle distributions}

Goudsmit and Saunderson developed a formulation for approximating the angle distributions for multiscattered electrons. These distributions are based on the number of collisions that occur and the average scattering angle per collision. The average scattering distribution for individual elastic collisions is described by a Legendre expansion, $P_{l}\left(\cos \theta_{1}\right)$. The addition theorem for spherical harmonics is invoked to determine the Legendre coefficients for electrons that have encountered $n$ collisions by assuming azimuthal symmetry for each scattering event. The final Legendre expansion coefficients, $G_{l}$, for the multiscatter angle distribution are obtained by a weighted average over the number of collisions encountered,

$G_{l}=\sum_{n=0}^{\infty} \psi_{n}(d)\left(P_{l}\left(\cos \theta_{1}\right)\right)_{\mathrm{ave}}^{n}$.

The normalized $n$th collided flux, $\psi_{n}(d)$, is given in terms of the actual path-length traveled, $d$, rather than

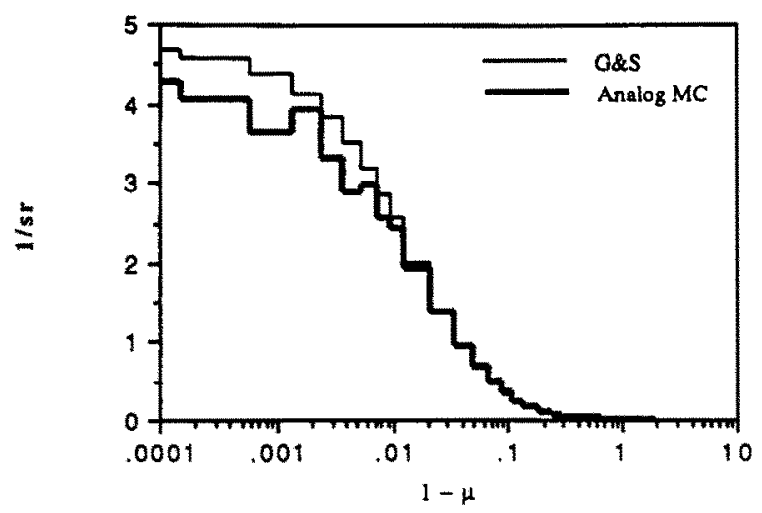

Fig. 7. Multiscatter angle distributions for $64 \mathrm{keV}$ electrons after a path-length of $8.91 \times 10^{-6} \mathrm{~cm}$ in gold.

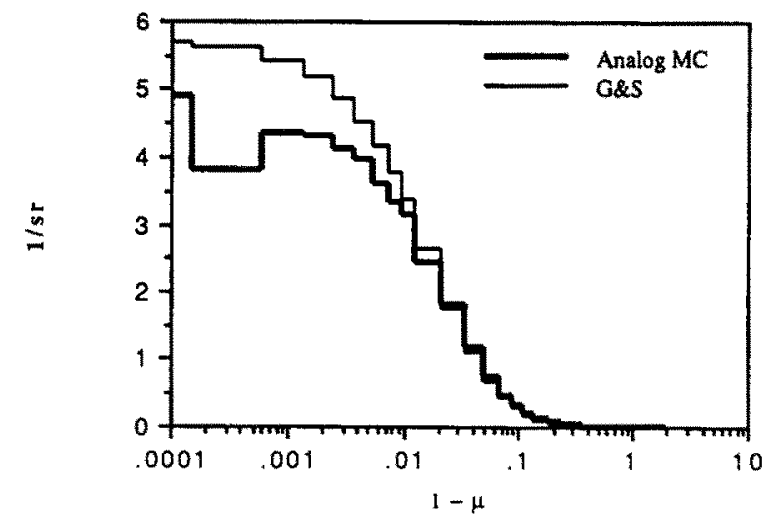

Fig. 8. Multiscatter angle distributions for $128 \mathrm{keV}$ electrons after a path-length of $3.18 \times 10^{-4} \mathrm{~cm}$ in aluminum.

the spatial position. This flux, the probability of undergoing $n$ collisions after traveling a path-length, $d$, is given by a Poisson distribution,

$\psi_{n}(d)=\frac{\mathrm{e}^{-\Sigma_{t} d}\left(\Sigma_{t} d\right)^{n}}{n !}$

A multiscatter angle distribution is constructed from the Legendre moments, $G_{l}$, in the usual way.

Goudsmit-Saunderson distributions used in MCNP4 were compared to equivalent distributions calculated using the analog Monte Carlo code. The multiscatter angle distributions for electrons with energies of $256 \mathrm{keV}$ and $64 \mathrm{keV}$ traveling through gold are shown in figs. 6 and 7 , respectively. Similar distributions were obtained in aluminum as shown in figs. 8 and 9 for energies of $128 \mathrm{keV}$ and $32 \mathrm{keV}$, respectively. Because electron scattering in gold is less forwardly peaked than in aluminum, the Goudsmit-Saunderson angular distribution is more accurate for gold. This superior accuracy reveals itself in the better agreement with the analog Monte Carlo results in figs. 6 and 7.

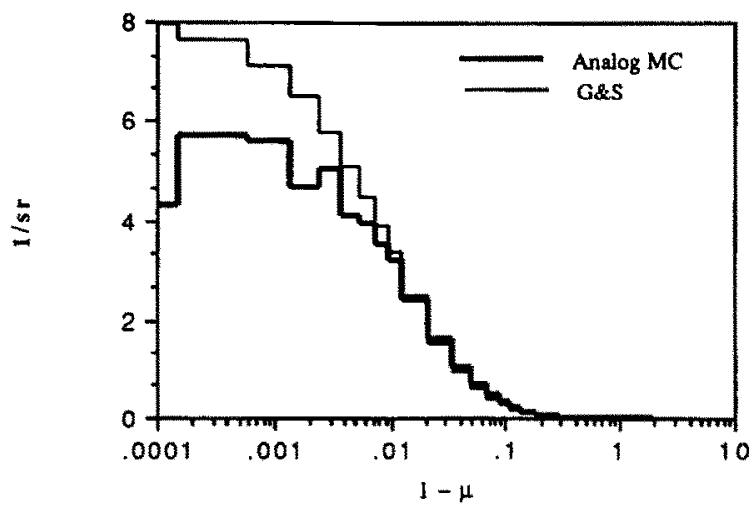

Fig. 9. Multiscatter angle distributions for $32 \mathrm{keV}$ electrons after a path-length of $3.17 \times 10^{-5} \mathrm{~cm}$ in aluminum. 


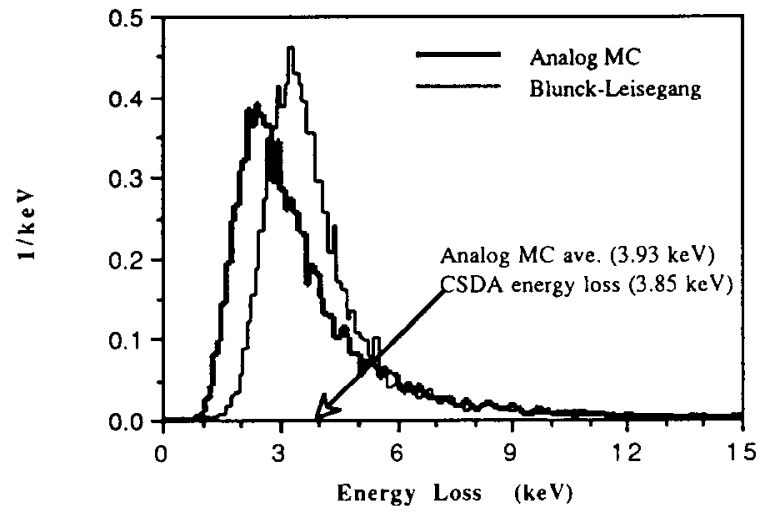

Fig. 10. Multiscatter energy distributions for $256 \mathrm{keV}$ electrons after a path-length of $7.82 \times 10^{-4} \mathrm{~cm}$ in aluminum.

\subsection{Multiscatter energy distributions}

The simplest energy loss formulation is given by the continuous slowing down approximation; the correct average energy loss is preserved but details about the random individual interactions are lost. Multi-scatter energy distributions (energy straggling distributions) were developed to account for the statistical nature of the energy loss for inelastic collisions. The multiscatterer energy distribution, developed by Landau [18] with correction by Blunck and Leisegang [19], is typically used in condensed history codes. Unlike Goudsmit and Saunderson's angular treatment, Landau's formulation is restricted to multiscattered electrons. The energy distributions represent electron travel over a full energy step instead of a substep to ensure that electrons undergo multiple collisions. The energy loss distribution for the substep distance is calculated by dividing the energy loss by the number of substeps. This normalization assumes that the shape of the distribution remains constant with distance, an assump-

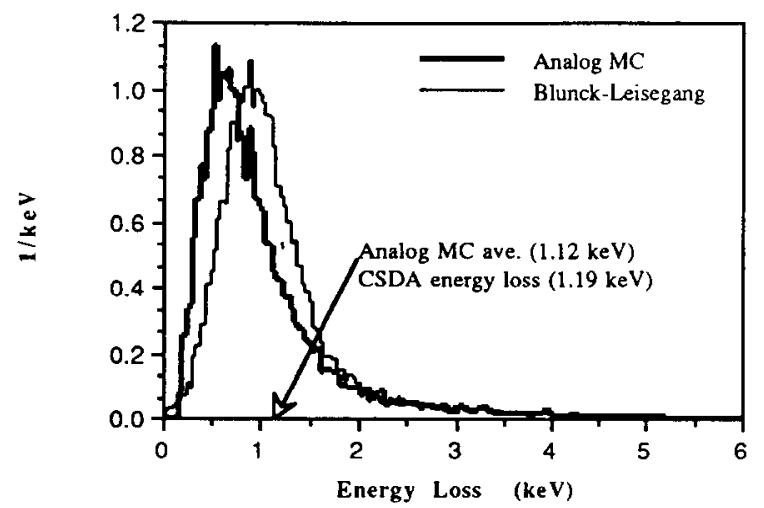

Fig. 11. Multiscatter energy distributions for $64 \mathrm{keV}$ electrons after a path-length of $1.03 \times 10^{-4} \mathrm{~cm}$ in aluminum.

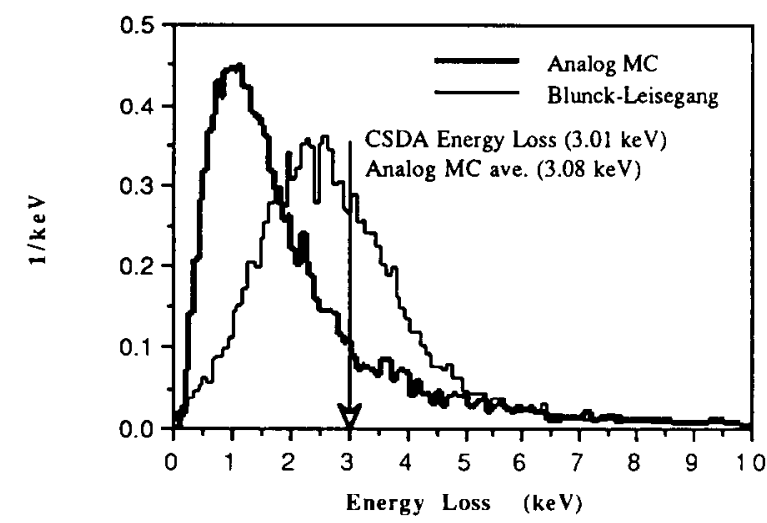

Fig. 12. Multiscatter energy distributions for $512 \mathrm{keV}$ electrons after a path-length of $1.46 \times 10^{-4} \mathrm{~cm}$ in gold.

tion which is obviously not true since low energy electrons lose a larger fraction of their energy in single collisions than higher energy electrons. The exponential increase in the stopping power with decreasing energy supports this assertion

Multiscatter energy distributions from MCNP4 are compared to distributions generated by the analog Monte Carlo code for electrons that travel a path-length equal to the substep. Results for electrons in aluminum with energies of $256 \mathrm{keV}$ and $64 \mathrm{keV}$ are shown in figs. 10 and 11 , respectively. Blunck-Leisegang theory disagreed with the analog Monte Carlo predictions. The theoretical treatment compared even worse with ana$\log$ Monte Carlo for electrons in gold as seen in figs. 12 and 13 for electrons with energies of $512 \mathrm{keV}$ and 32 $\mathrm{keV}$ respectively. The distributions by Blunck and Leisegang are always wider and peak at a higher energy loss than the analog Monte Carlo results. The analog Monte Carlo distributions have long, low probability tails for large energy losses yet the average values of the distributions are always close to the CSDA

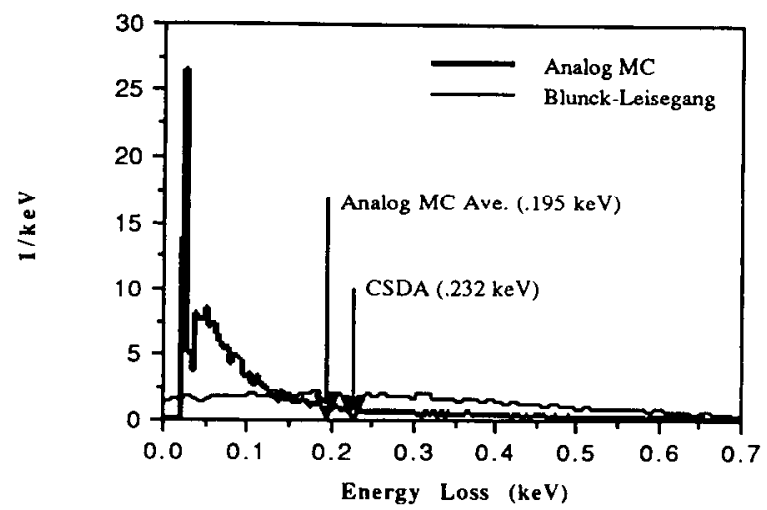

Fig. 13. Multiscatter energy distributions for $32 \mathrm{keV}$ electrons after a path-length of $2.87 \times 10^{-6} \mathrm{~cm}$ in gold. 


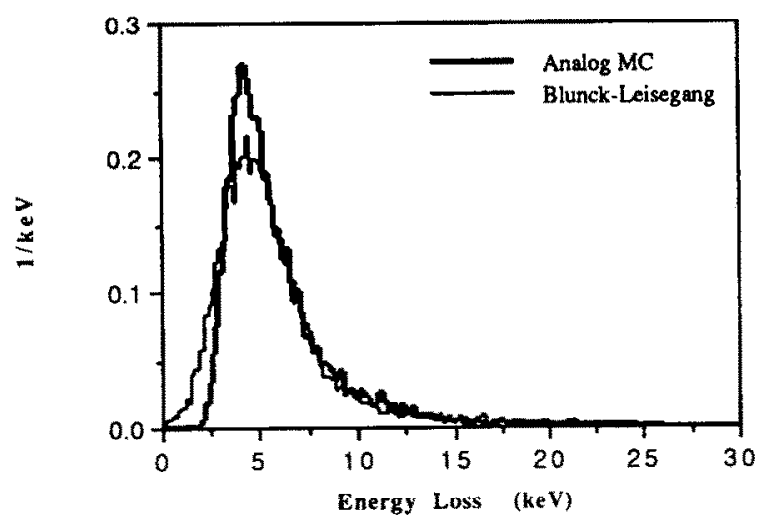

Fig. 14. Multiscatter energy distributions for $64 \mathrm{keV}$ electrons after a path-length of $5.17 \times 10^{-4} \mathrm{~cm}$, an energy step, in aluminum.

predictions except for the $32 \mathrm{keV}$ electrons in fig. 13 . This is due to the large percentage of electrons that have escaped without an interaction since the CSDA is based on the average energy loss for collided particles.

These comparisons were performed over default substep sizes used in MCNP4. Blunck-Leisegang distributions were derived for full energy step sizes then normalized to substeps distances. The above comparisons reveal the inaccuracies in the distributions as they are implemented in the condensed history code. Additional comparisons were made for energy loss distributions over a full energy step. Blunck-Leisegang and Monte Carlo energy distributions show better agreement over a full energy step than they did over a substep as seen in figs. 14 and 15. Blunck and Leisegang's theory is inapplicable for low energy electrons, espccially in high $Z$ matcrials. Not only arc there inaccuracies with the Blunck-Leisegang theory but the substep normalization process is in error.

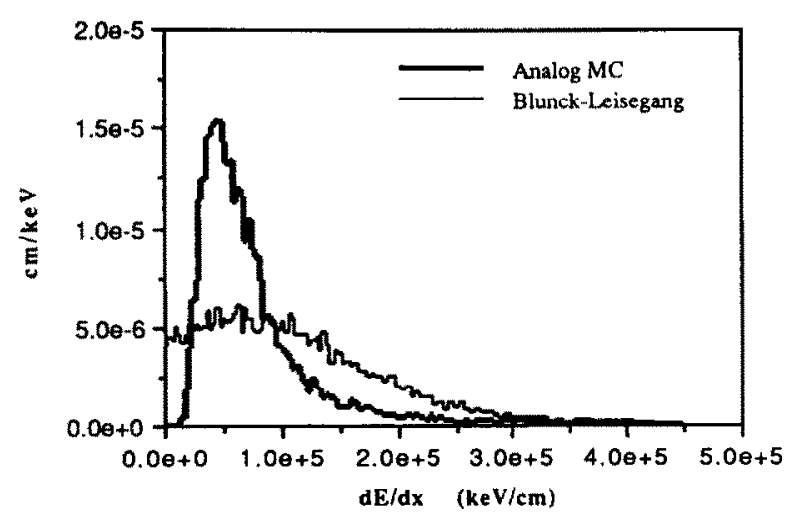

Fig. 15. Multiscatter energy distribution for $32 \mathrm{keV}$ electrons after a path-length of $3.73 \times 10^{-5} \mathrm{~cm}$, an energy step, in gold.

\section{Replacement of the multiscatter distributions}

Many analytical and empirical corrections can be made to condensed history through changes in the multiscatter distributions to improve the results for low energy problems. It is not clear whether the altered multiscatter distributions constitute a real improvement or simply an artifact from error cancelation. The previous comparisons show the error in the theoretically generated energy and direction PDFs. It is difficult to determine the impact that the deviation has on the final transport results by simple comparing the PDFs. As a means of evaluating this impact, the multiscattering PDFs used in the condensed history code MCNP4 were replaced with multiscatter distributions generated by the single-scatter Monte Carlo code. The results obtained represent the best a condensed history type method can achieve since the distributions are exact to within statistical fluctuations of the analog Monte Carlo calculation.

The modified version of MCNP4 samples from these new PDFs for angle and energy (thus the name MCNP4AE). The analog generated PDFs were constructed by tracking 100000 electron histories and recording the energy and direction after traveling a distance equal to an MCNP4 defaull substep size. MCNP4AE results are relatively unchanged from MCNP4 calculations for backscatter calculations in aluminum (fig. 16). Results where the energy loss was calculated by the CSDA in the two codes (MCNP4 and MCNP4AE), shown in fig. 17, further isolate the changes induced by using replacement PDFs. The close agreement in these comparisons shows that the energy and angle distributions used in condensed history for aluminum agree fairly well with analog distributions. Similar analysis was performed for transport through gold with a much different outcome as seen in figs. 18 and 19. The close agreement between MCNP4 and

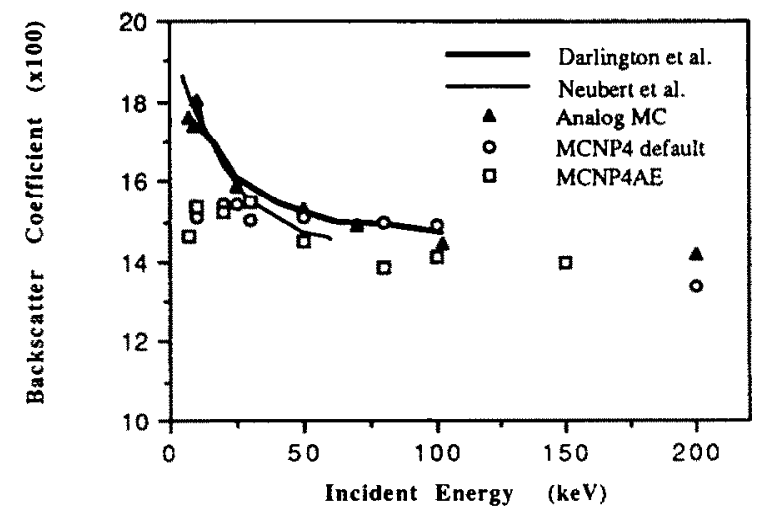

Fig. 16. Backscatter as a function of energy using the energy loss distributions in aluminum. 


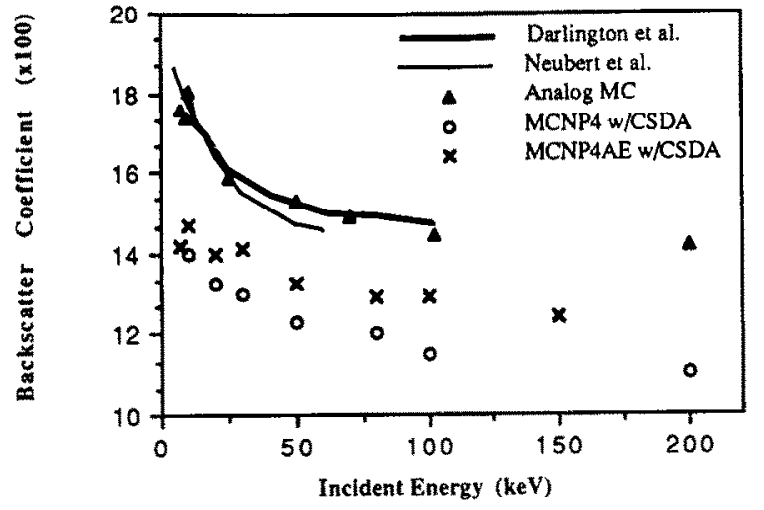

Fig. 17. Backscatter as a function of energy using the CSDA for energy loss in aluminum.

MCNP4AE when the CSDA was used for the energy loss suggest that the energy treatment rather than the angular distributions are incorrect. Backscatter results were dramatically improved for energies below 100 $\mathrm{keV}$ in gold when both the analog generated angle and energy distributions were used.

\section{Discussion}

Of the three modes of failure mentioned in section 1 , inaccuracies in the multiscatter energy loss distributions impacts the condensed history results most significantly. The results reveal that Blunck-Leisegang distributions are unsatisfactory even though they preserve the correct average energy loss. Agreement with experiment improved when the conventional energy loss straggling PDFs were replaced by PDFs generated from analog Monte Carlo especially for transport in gold below $100 \mathrm{keV}$. The backscatter coefficient remained relatively unchanged in aluminum with these

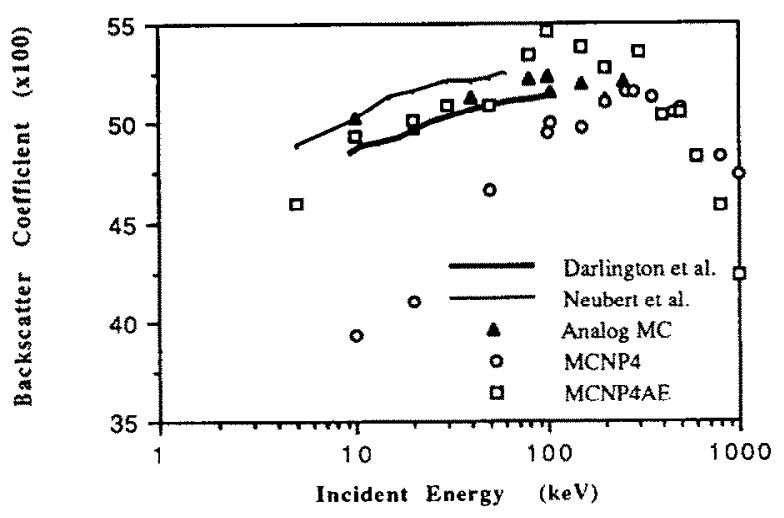

Fig. 18. Backscatter as a function of energy using the energy loss distributions in gold.

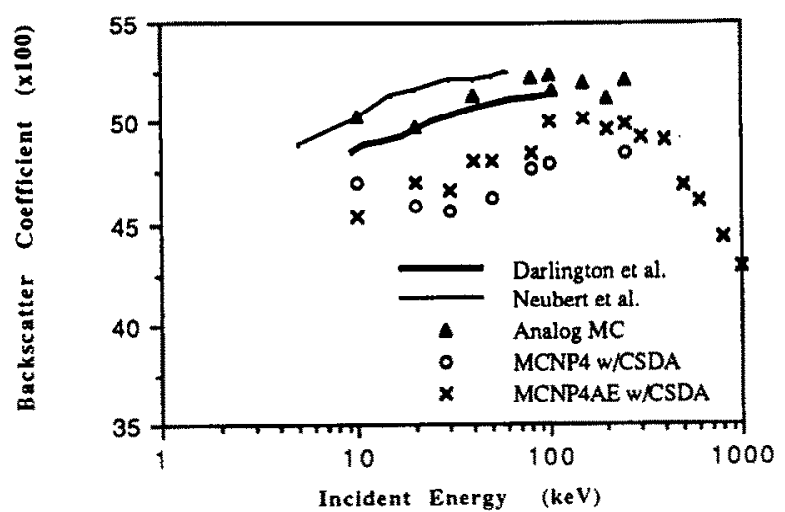

Fig. 19. Backscatter as a function of energy using the CSDA for energy loss in gold.

replacements. The Blunck-Leisegang straggling distributions are biased towards higher energy loss per substep which results in an under-prediction of the backscatter percentage.

The multiscatter angle astributions as given by Goudsmit and Saunderson's theory agree fairly closely with distributions generated via analog Monte Carlo. Insignificant differences were found when MCNP4 was compared to MCNP4AE using the CSDA for energy loss. This comparison isolated the multiscatter angle distribution by holding all other variables constant and proved that even the "correct" angle distributions will not give better results.

Even with the correct angle and energy distributions, the condensed history method does not faithfully reproduce the analog Monte Carlo results. This difference is attributable to the straight-line approximation used in condensed history. Even with the addition of path-length and lateral displacement corrections suggested by Berger [1], results were not always improved. A new method, Response History Monte Carlo

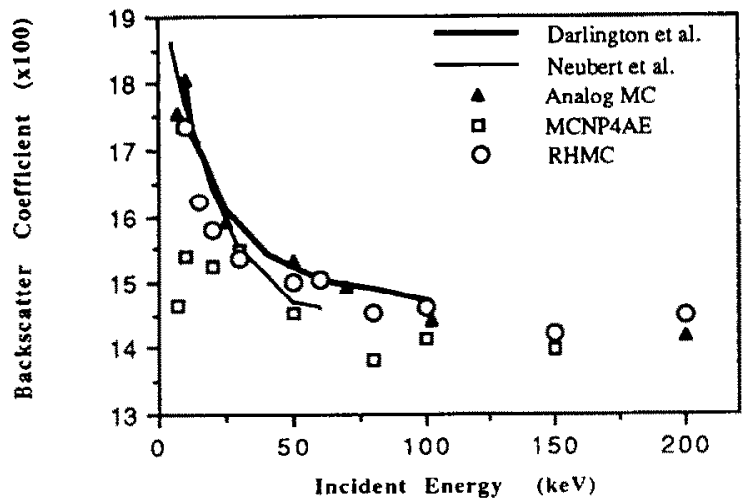

Fig. 20. Backscatter as a function of incident energy for normally incident electrons on thick aluminum. 


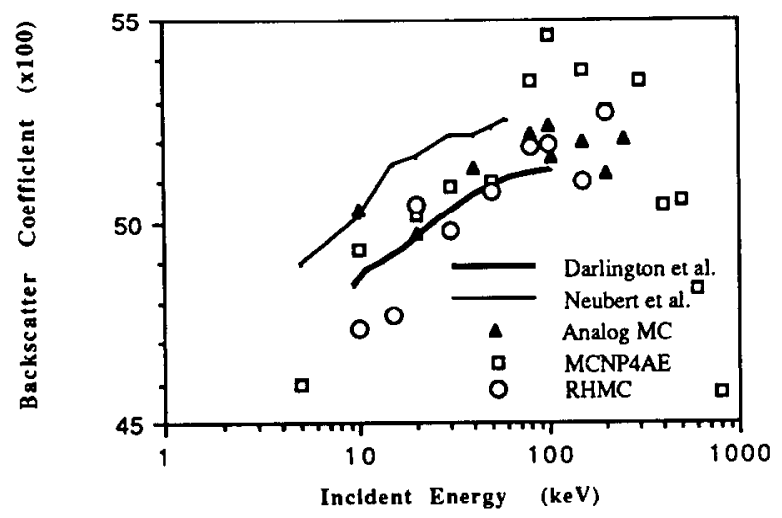

Fig. 21. Backscatter as a function of incident energy for normally incident electrons on thick gold.

(RHMC) [20], has been developed to correct for the trajectory butchering done in condensed history codes. This method shows closer agreement with analog Monte Carlo (figs. 20 and 21). The RHMC method uses distributions representing multiscattered electrons to step through a material with an additional spatial PDF to account for the electron position at the end of the substep. Energy and angle distributions are coupled with position to give a more accurate description of the electron trajectory in a calculation. Single-scatter Monte Carlo results have been reproduced in a fraction of the computational time using this more detailed treatment of the electron trajectory.

To extend the validity of the condensed history method, improvements must be made in

(1) the energy loss straggling distributions, and

(2) corrections to the straight-line approximation.

Substantially better results were observed in gold by replacing the conventional energy loss PDFs with more accurate PDFs generated by a single-scatter Monte Carlo code. Calculations for transport in aluminum showed little change using the analog generated PDFs. Results were improved in both materials using the more detailed spatial and energy treatment in the RHMC method.

\section{References}

[1] M.J. Berger, Meth. Comput. Phys. 1 (1963) 135.

[2] C.T. Ballinger, J.A. Rathkopf, and W.R. Martin, The Response Matrix Monte Carlo Method Based on a General Geometry Local Calculation for Electron Transport, Proc. Advances in Mathematics, Computations, and $\mathrm{Re}$ actor Physics, American Nuclear Society, Vol. 5 (1991).

[3] D.E. Cullen and S.T. Perkins, The Livermore Bremsstrahlung Data Base, Lawrence Livermore National Laboratory, Livermore, CA, UCID-21627 (1989).

[4] S.T. Perkins and D.E. Cullen, The Livermore Electron Impact Ionization Data Base, Lawrence Livermore National Laboratory, Livermore, CA, UCID-21628 (1989).

[5] S.T. Perkins and D.E. Cullen, The Livermore Electron Elastic Scattering Data Base, Lawrence Livermore National Laboratory, Livermore, CA, UCRL-ID-103170 (1990).

[6] N.F. Mott, Proc. Roy. Soc. A126 (1930) 259.

[7] M.E. Riley, C.J. McCallum, and F. Biggs, At. Data and Nucl. Data Tables (1983) 28, 379.

[8] J.C. Ashley, C.J. Tung, and R.H. Ritchie, Surf. Sci. 81 (1978) 409.

[9] M.J. Berger and S.M. Seltzer, Stopping powers and ranges of electrons and positrons, U.S. Dept. of Commerce, National Bureau of Standards, NBSIR 82-2550 (1982).

[10] E.H. Darlington, J. Phys. D8 (1975) 85.

[11] H. Kanter, Ann. Physik 20 (1957) 144.

[12] D.H. Rester and J.H. Derrickson, J. Appl. 42 (2) (1971) 714.

[13] MCNP4 - A gencral Monte Carlo Code for neutron, photon, and electron transport, LANL 7936-M Rev. 4 (1991).

[14] A.J. Antolak and W. Williamson, Jr., Electron backscattering from bulk materials, SAND84-8969 (1985).

[15] G. Neubert and S. Rogaschewski, Phys. Status Solidi (a) 59 (1980) 35 .

[16] G. Molière, Z. Naturforsch. 2a (1947) 133.

[17] S. Goudsmit and J.L. Saunderson, Phys. Rev. 57 (1940) 24.

[18] L. Landau, J. Phys. USSR 8 (4) (1944) 201.

[19] O. Blunck and S. Leisegang, Z. Physik 128 (1950) 500.

[20] C.T. Ballinger, The Response History Monte Carlo Method for Electron Transport, Ph.D. thesis, University of Michigan (1991). 